\title{
Detection of alpha-fetoprotein through biological signal amplification by biosensor based on imaging ellipsometry
}

\author{
Chenghong Huang a,c,d, Yanyan Chen a,*, Chunxia Wang a, Wei Zhu ${ }^{\mathrm{e}}$, Hongwei Ma ${ }^{\mathrm{a}}$, Gang Jin ${ }^{\mathrm{a}, \mathrm{b}, \mathrm{c}, *}$ \\ a Suzhou Institute of Nano-tech and Nano-bionics, Chinese Academy of Sciences, Suzhou, China \\ ${ }^{\mathrm{b}}$ NML, Institute of Mechanics, Chinese Academy of Sciences, Beijing, China \\ c Institute of Biophysics, Chinese Academy of Sciences, Beijing, China \\ ' Graduate School of the Chinese Academy of Sciences, Beijing, China \\ e Radiation Medical Institute of Shandong Academy of Medical science, Jinan, China
}

\section{A R T I C L E I N F O}

Available online 8 December 2010

\section{Keywords:}

Imaging ellipsometry

Biosensor

Signal amplification

Alpha-fetoprotein

Hepatocellular carcinoma

\begin{abstract}
A B S T R A C T
High sensitive and quantitative detection of alpha-fetoprotein (AFP) by biosensor based on imaging ellipsometry (BIE) through biological amplification was investigated. AFP firstly reacted with the rat monoclonal antibody (rat-mAb) initially immobilized on glutaraldehyde modified silicon surface, then rabbit anti-human AFP polyclonal antibodies (Rabbit-pAb) and goat anti-rabbit IgG (goat-IgG) were sequentially applied to amplify signal. Results revealed that signal was enhanced approximately six fold. The linear range of AFP detection was $20.0-200.0 \mathrm{ng} / \mathrm{ml}$ with a low limit of $5.0 \mathrm{ng} / \mathrm{ml}(\mathrm{S} / \mathrm{N}=3)$. The cross-reaction rate was less than $5.2 \%$ evaluated by biomarker (carcinoembryonic antigen, carbohydrate 19-9 and carbohydrate antigen 242) and two common proteins (human serum albumin, fibrinogen) and their mixture. Coefficient variation (CV) for intra-slide and inter-slide reproducibility were $10.3 \%, 6.6 \%, 6.3 \%$ and $10.7 \%, 7.9 \%, 6.4 \%$ for $41.6 \mathrm{ng} / \mathrm{ml}, 83.2 \mathrm{ng} / \mathrm{ml}$ and $128.4 \mathrm{ng} / \mathrm{ml} \mathrm{AFP}$. In clinic application, cut-off value at $28.6 \mathrm{ng} / \mathrm{ml}$ was also determined with sensitivity 0.72 and specificity 0.94 . Results of 47 clinic patient samples detected by BIE were in good agreement with those of electrochemiluminescence immunoassay (ECLIA) (R2=0.9949).The area under receiver operating characteristic curve (ROC) curve is 0.89. Statistical analysis showed that BIE is high agreement with ECLIA (Kappa $=0.733, \mathrm{U}=3.21>\mathrm{U} 0.01$ ). It shows a potential for hepatocellular carcinoma (HCC) diagnosis.
\end{abstract}

(c) 2010 Elsevier B.V. All rights reserved.

\section{Introduction}

Biomarkers can offer much more information for identification of early cancer and people at risk of developing cancer[1]. Alpha-protein (AFP), associated with hepatocellular carcinoma (HCC), is normally produced by both yolk sack and fetal liver during neonatal development, but it abruptly decreases soon after the birth - by the end of the second month postpartum, only a trace amount can be detected[2]. Now, AFP has already approved by FDA for HCC auxiliary diagnosis [3]. Therefore, sensitive detection and accurate analysis of AFP is very important for HCC monitoring and management.

Conventional immunoassays including radioimmunoassay(RIA) [4], enzyme-linked immunosorbent assay(ELISA) [5] and electrochemiluminescent immunoassay(ECLIA) [6] have inherent shortcomings such as partial activity loss of labeled antibody, radiation hazards and contaminations to environment. Furthermore, they also have some

\footnotetext{
* Corresponding authors. Jin is to be contacted at Tel.: +86 1082544318.

E-mail addresses: yychen2006@sinano.ac.cn (Y. Chen), gajin@imech.ac.cn (G. Jin)
}

limitations including long analysis time, requirement of skillful operators and single sample analysis. Therefore, development of sensitive, reliable, high-throughput techniques are now attracting significant attentions [7]. Compared with above methods, biosensor based on imaging ellipsometry (BIE)[8] has the advantages of labelfree, rapid, and high through-put analysis[9-11]. It has many applications especially in biomedical field [12-14].

In this study, immunoassay for AFP by BIE was investigated and the configuration was illustrated in Fig. 1. A sandwich-type strategy that AFP was firstly captured by rat anti-AFP monoclonal antibodies (rat-mAb) and then rabbit anti-AFP polyclonal antibody (rabbit-pAb) and goat anti-rabbit IgG (Goat $\operatorname{IgG}$ ) were sequentially applied to realize biological amplification for acquisition of high sensitivity and broad detection range was adopted. Because there were no validation guideline for biomarkers and protein array, the reproducibility of the immunoassay was evaluated using the Food and Drug Administration (FDA) regulatory guidelines for pharmacokinetic assay validation [16]. A spike-recovery test was used for accuracy assessment. For clinic application, cut-off value and receiver operating characteristic curve (ROC) by testing of serum sample were also performed. A systematic methodology was successfully established. 


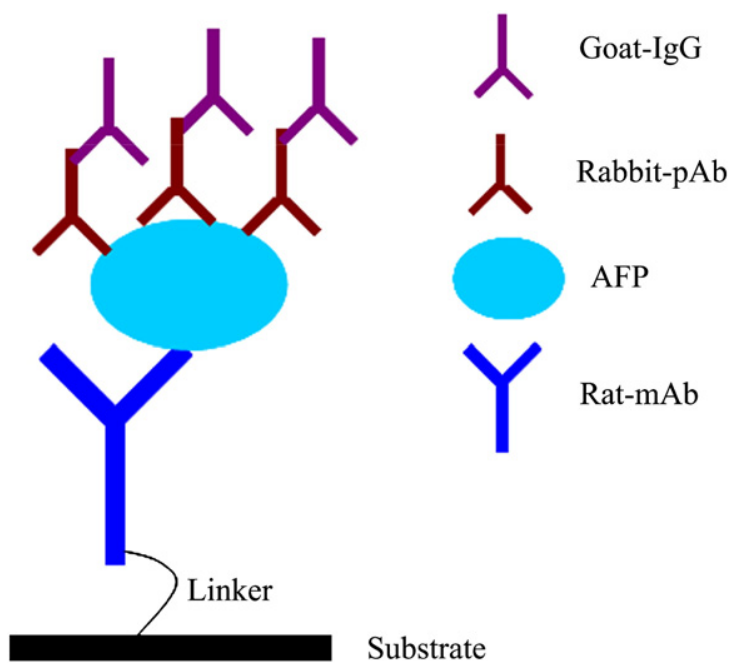

Fig. 1. The configuration of sandwich immunoassay for AFP detection.

\section{Materials and methods}

\subsection{Materials}

Silicon wafers were from General Research Institute for Nonferrous Metals (China). AFP and rat anti-AFP monoclonal antibody (rat$\mathrm{mAb}$ ) were purchased from Meridian Life Science, Inc. (USA). Rabbit anti-AFP polyclonal antibody (Rabbit-pAb) and goat-IgG were obtained from Beijing Kangwei Co. (China). Standard was obtained from National Institution for the Control of the Pharmaceutical and Biological Products (China). Carcinoembryonic antigen (CEA), carbohydrate antigen 19-9 (CA19-9) and carbohydrate antigen 242 (CA242) were all purchased from Shanghai Linc-Bio Science Co. (China). Fetal bovine serum (FBS) was purchased from Beijing Dingguo Co. (China). Human serum albumin (HSA), fibrinogen (Fib), 3-aminopropyltriethoxysilane (APTES) and glutaraldehyde (GA, 50\%aqueous solution) were obtained from Sigma-Aldrich. Water was obtained from a millipore Milli-Q ion exchange apparatus. Phosphate-buffered saline (PBS, $8 \mathrm{mM} \mathrm{Na} 2 \mathrm{PO} 4 \cdot 2 \mathrm{H} 2 \mathrm{O}, 2.68 \mathrm{mM} \mathrm{KCl}$, 1.14 Mm KH2PO4, $137 \mathrm{mM} \mathrm{NaCl}$; pH 7.4) was prepared in deionized water.

\subsection{BIE principle}

BIE presented here has been developed in Institute of Mechanics (Chinese Academy of Sciences, China) for performing parallel immunoassays, which is a combination of an integrated microfluidic array system and imaging ellipsometry (IE)[16]. The microfluidic array system is used for surface patterning and array fabrication, solution delivery, antibody immobilization and antigen capture. IE is used for reading the protein array.

The polydimethylsiloxane (PDMS) template in microfluidic array system contains a $8 \times 6$ cell array. When the cell array was attached to the silicon wafer surface, 48 individual chambers were formed independently. As each chamber had two access holes, namely, an inlet and an outlet, protein solution could transfer in and out of the chamber. By such microfluidic system, protein solutions were delivered individually to different cells for immobilization so that the surface is patterned simultaneously in array format. Accordingly, multiple protein dots were patterned regularly on substrate and the physical size of each dot is approximately $1.5 \mathrm{~mm} \times 1.0 \mathrm{~mm}$.

IE is a display technique for ultra-thin film and surface characterization. As an enhancement of traditional ellipsometry, IE used a CCD camera to image the ellipsometry response of a larger area sample, and the result was grabbed as a digital image and stored in a computer with a grayscale format (8 bits, 0-255 grayscale) for further evaluation by an image-processing program. Once imaging ellipsometer was fixed, the detected signal intensity "I" (grayscale) is the function of the layer thickness $(d), I=f(d)$, where $f(d)$ denoted the function relationship which is determined by layers with known intensity and thicknesses, then the unknown thickness of protein layer could be deduced from the detected intensity according to the function. At the same time there is a relationship between surface concentration and layer thickness: surface concentration ( $\mu \mathrm{g} /$ $\left.\mathrm{cm}^{2}\right) \approx \mathrm{K} \times \mathrm{d}(\mathrm{nm})$, where $\mathrm{K}=0.12$ [17]. Thus, the grayscale value directly reflects layer thickness and surface concentration. The higher the grayscale value, the thicker the layer and the higher the surface concentration. Here the angle of incidence is 75 and the optical wavelength is $632.8 \mathrm{~nm}$ [18].

\subsection{Silicon wafer treatment}

Silicon treatment was referred to literature [19]. Silicon wafers were immersed in piranha solution $\left(\mathrm{H}_{2} \mathrm{SO}_{4}: \mathrm{H}_{2} \mathrm{O}_{2}=3: 1, \mathrm{v} / \mathrm{v}\right)$ to oxidize $30 \mathrm{~min}$ followed by intensive rinsing with deionized water. The oxidized silicon wafer were then treated with APTES in ethanol solution $(1: 15, v / v)$ for $2 \mathrm{~h}$, washed by ethanol and PBS repeatedly, then placed in glutaraldehyde/PBS solution $(10: 1, \mathrm{v} / \mathrm{v})$ for $1.5 \mathrm{~h}$, and washed by PBS several times again. The treated silicon wafers were immediately used or placed in PBS at $4{ }^{\circ} \mathrm{C}$ for later use.

\subsection{Antibody immobilization}

Rat-mAb as capture antibody were diluted by PBS prior to use, then, dispensed into each channel by microfluidic system and immobilized on the glutaraldehyde silicon surface at $1 \mu \mathrm{l} / \mathrm{min}$ for $20 \mathrm{~min}$. In this step, the amino group of antibody molecule will covalently attached with the aldehyde group on silicon substrate. After immobilization, $\operatorname{PBST}(0.05 \%)$ was used to wash all of the channels at $20.0 \mu \mathrm{l} / \mathrm{min}$ for $5 \mathrm{~min}$. Then, $1 \mathrm{M}$ ethanolamine was dispensed to deactivate the unbound aldehyde group at $5.0 \mu \mathrm{l} / \mathrm{min}$ for $10 \mathrm{~min}$ and washed by PBST $(0.05 \%$ tween-20) again at $20.0 \mu \mathrm{l} / \mathrm{min}$ for $5 \mathrm{~min}$. Fetal bovine serum (FBS, 1:10 dilution) was subsequently dispensed to block the surface at $1.0 \mu \mathrm{l} / \mathrm{min}$ for $30 \mathrm{~min}$. The channels were washed by PBST, and sensing layer for AFP detection was achieved.

\subsection{Detection of AFP and clinic serum application}

Initially, AFP solution (standard or serum sample) was flowed through channel at $1.0 \mu \mathrm{l} / \mathrm{min}$ for $20 \mathrm{~min}$. This process permits capture antibody of sensing layer to catch target antigen in sample specifically. PBST was then used to wash at $20.0 \mu \mathrm{l} / \mathrm{min}$ for $5 \mathrm{~min}$. Next, rabbit-pAb was added to react with AFP at $1.0 \mu \mathrm{l} / \mathrm{min}$ for $15 \mathrm{~min}$ and PBST was used to wash at $20.0 \mu \mathrm{l} / \mathrm{min}$ for another $5 \mathrm{~min}$. Finally, goat-IgG was dispensed to combine with Rabbit-pAb at $1.0 \mu \mathrm{l} / \mathrm{min}$ for $15 \mathrm{~min}$. After all channels were washed by PBST and deionlized water repeatedly at $20.0 \mu \mathrm{l} / \mathrm{min}$ for $5 \mathrm{~min}$, the silicon wafer was taken from the PDMS template, dried under a stream of nitrogen and delivered to imaging ellipsometry for grayscale measurement. The total assay time per slide was approximately $60 \mathrm{~min}$ and all manipulations were accomplished at room temperature.

\subsection{Statistical analysis}

Parametric results of healthy and patient group were expressed as mean \pm standard deviation (SD) and difference between groups was compared by independent-samples Test. Non-categorical data were compared by chi-squared test. Comparison between BIE and ECLIA was made using bivariate Spearman's correlation test ( $\mathrm{P}$ P value $<0.05$ was considered as statistically significant). The agreement was achieved by kappa analysis. 


\section{Results}

\subsection{Optimization of reaction conditions}

Capture antibody concentration was firstly screened. Serial diluted capture antibodies were dispensed to the glutaraldehyde modified silicon surface for immobilization. Saturation immobilization was arrived at $120.0 \mu \mathrm{g} / \mathrm{ml}$ upward. Furthermore, there was a significant difference in efficacy of different capture antibody concentrations to combine $25.0 \mathrm{ng} / \mathrm{ml} \mathrm{AFP} \mathrm{(Cut-off} \mathrm{value} \mathrm{for} \mathrm{distinguishing} \mathrm{normal} \mathrm{or}$ abnormal) and $100.0 \mathrm{ug} / \mathrm{ml}$ has maximum antigen binding capacity. Whatever higher or lower this concentration is not propitious to grayscale response. This can be interpreted as high concentration of capture antibody is unfavorable for antigen accessible due to steric hindrance, and low concentration is scarce of enough sites.

Concentration of rabbit-pAb and goat-IgG were subsequently optimized. After AFP with concentration of 20.0, 40.0, 80.0, 120.0 and $160.0 \mathrm{ng} / \mathrm{ml}$ was added to combine with capture antibody, different concentrations of rabbit-pAb was added to combine with captured AFP and goat-IgG was delivered to combine with rabbit-pAb for optimal dose-response, as well as best detection limit. Considering that there is a trade-off between the maximal linear detection limit of CCD $(<230)$ and the broader dynamic range of AFP, as well as a higher sensitivity preferred, the optimum concentrations of rabbit-pAb and goat-IgG were determined as 1:20 and 1:30, respectively.

\subsection{Efficiency of signal amplification}

To gain further insight into efficiency of signal amplification, we elaborately compared the strategies of without amplification, primary amplification (only rabbit-pAb was applied for amplification) and secondary amplification (rabbit-pAb and goat-IgG were sequentially applied for amplification), and grayscale image results were shown in Fig. 2 (a), (b) and (c), respectively. In these grayscale images, six dots in each column were corresponding to different AFP concentration $(0$, 20.0, 40.0, 80.0, 120.0 and $160.0 \mathrm{ng} / \mathrm{ml}$ ), and each row was duplicates. Curves of grayscale value vs. AFP concentration are for the three strategies (Fig. 3). It is obvious that secondary amplification (strategy c) can provide maximum signal compared with the other two (strategy a and b). According to calculation, the signal was amplified approximately six fold in strategy c, which predicted that AFP can bind about three rabbit-pAb molecules after it had been bound with the capture antibody.

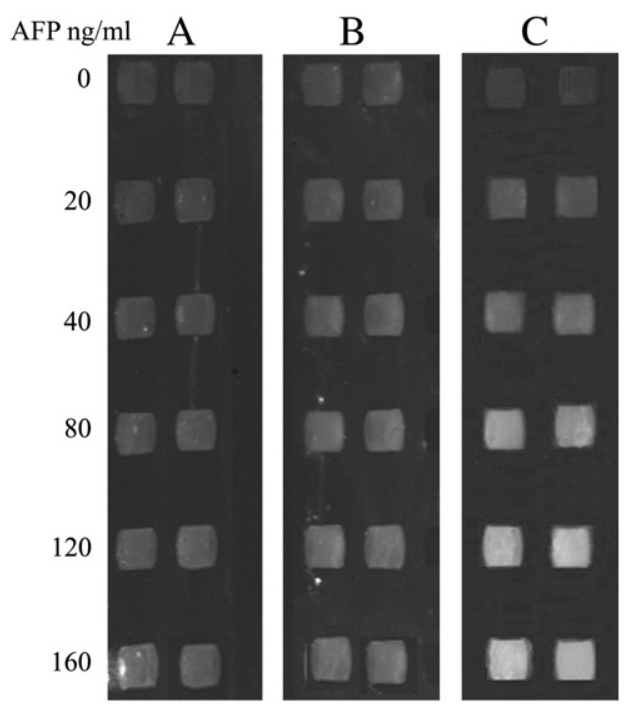

Fig. 2. Typical ellipsometric images for AFP detection with three detection strategy. (A) without amplification, (B)primary amplification and (C)secondary amplification. Each strategy is conducted duplicates.

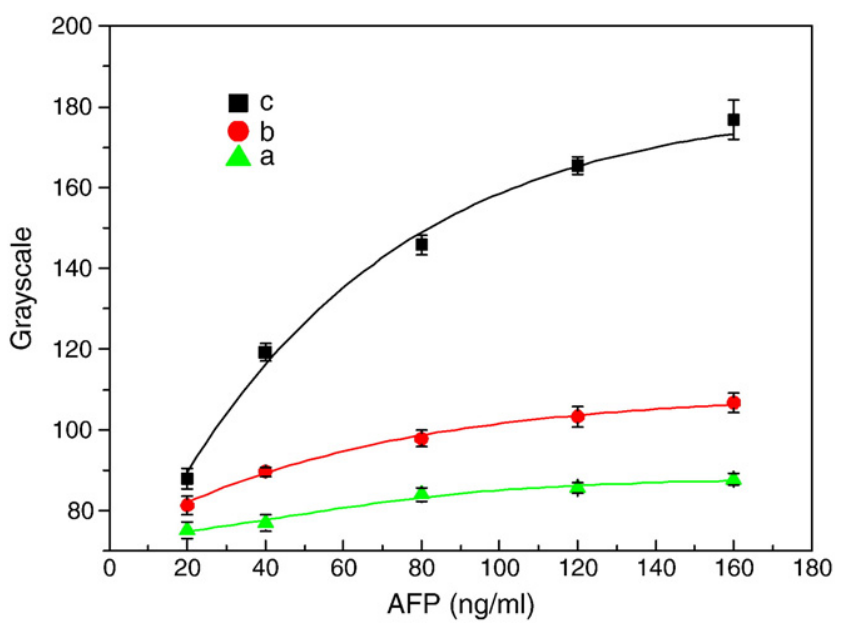

Fig. 3. Efficiency of signal amplification. Three strategies including without amplification ( $\mathbf{\Delta})$, primary amplification $(\bullet)$ and secondary amplification $(\boldsymbol{\square})$ for AFP detection, where a denotes only AFP addition, $\mathrm{b}$ denotes rabbit-pAb applied and c denotes rabbit$\mathrm{pAb}$ and goat-IgG sequentially applied after AFP addition, are illustrated.

This is basically consistent with the results acquired by Teramura et al [20] when surface plasmon resonance was performed.

\subsection{Dose-response, calibration curve and sensitivity}

Dose-response of AFP was performed and results were shown in Fig. 4. The representative ellipsometric image in Fig. 4 (A) shows that the grayscale was increased with the inc rease of AFP concentration. The grayscale could be also transferred into three-dimension distribution for intuitionistic observation, shown in Fig. 4(C). Mean grayscale value from three independent tests vs. AFP concentrations was plotted in Fig. 5. By four-parameter logistic function fitting, the curve can be represented by the regression equation: $\mathrm{Y}=-29.3+$ 93.6log $\times(R 2=0.989, P<0.01)$, where $Y$ denotes the grayscale value and $\mathrm{x}$ is the logarithmic transformation of AFP concentration $(\mathrm{ng} / \mathrm{ml})$. By this equation, the minimum detection limit as low as $5 \mathrm{ng} / \mathrm{ml}(\mathrm{S} /$ $\mathrm{N}=3$ ) was acquired. There is a good linear relationship in the AFP

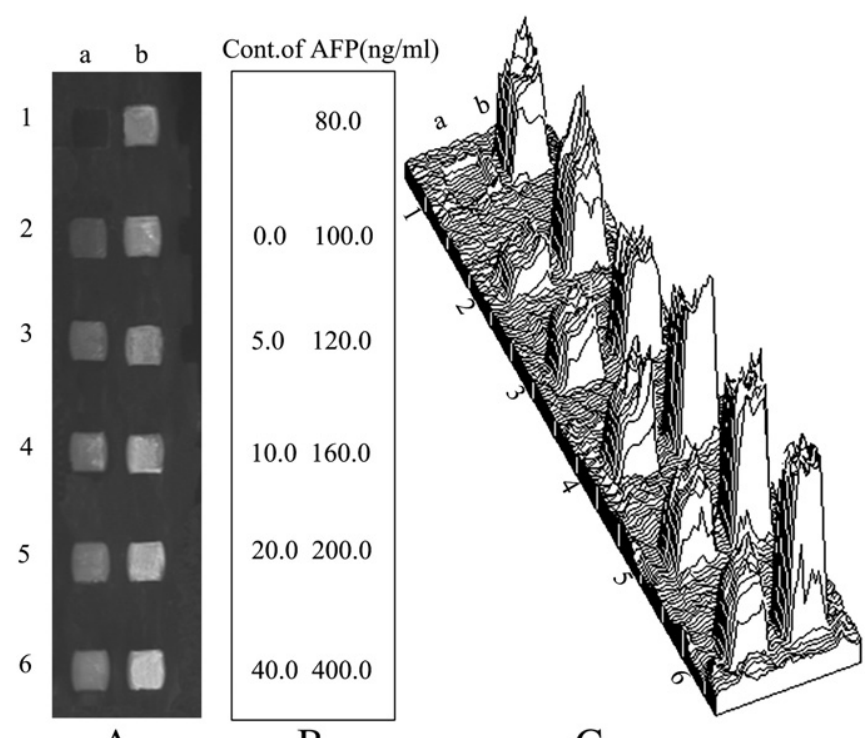

A

B

Fig. 4. Typical dose-response grayscale image (A),corresponding AFP concentration (B) and its three dimension map (C). Dot a1 is substrate control. Dots a2-b6 are AFP application with $0,5.0,10.0,20.0,40.0,80.0,100.0,120.0,160.0,200.0$ and $400.0 \mathrm{ng} / \mathrm{ml}$, respectively. 


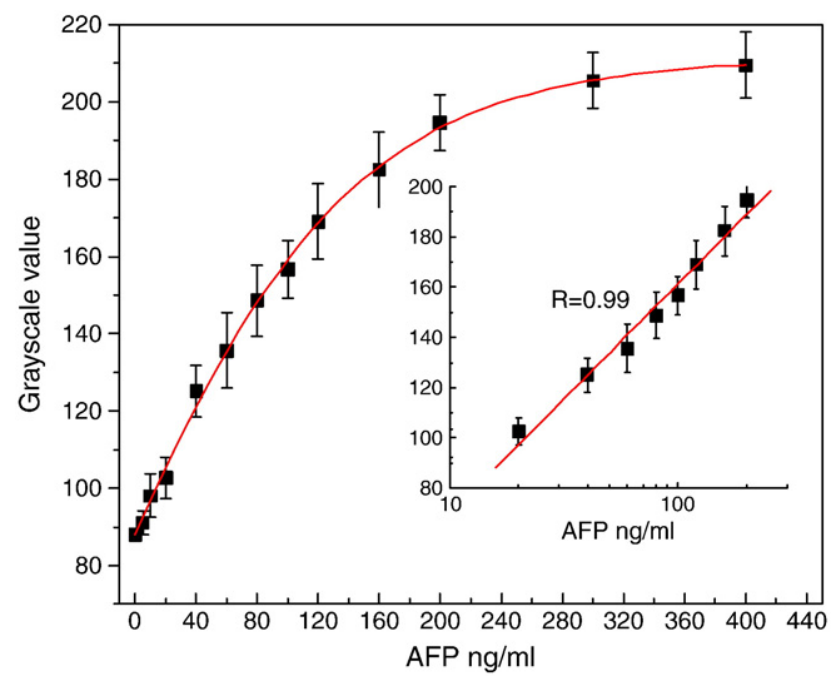

Fig. 5. Calibration curve of grayscale vs. AFP concentration. The data are the average value of three independent measurements. Inset shows linear detection range.

concentration range $20-200 \mathrm{ng} / \mathrm{ml}$ shown as an inset, which can be used as a standard curve for further quantitative analysis. By linear fitting, the linear relationship is obtained as $\mathrm{Y}=-21.8+91.6 \times$ $(\mathrm{R} 2=0.989)$.

\subsection{Reproducibility, accuracy and selectivity}

The reproducibility of the immunoassay was assessed by intraslide and inter-slide coefficients of variation (CV)[15,21]. CV\% was defined as $\mathrm{CV}=\sigma / x \times 100 \%$, whereois standard deviation and $x$ is mean value. In this work, the intra-slide CV\% were conducted by 8 tests for the same concentration AFP on one silicon slide and interslide CV\% were conducted by 5 tests for the same concentration on different silicon slides. The results were shown in Table 1 . The intraslide and the inter-slide CV\% obtained for AFP concentration of 41.6, 83.2 and $128.4 \mathrm{ng} / \mathrm{ml}$ were $10.3 \%, 6.6 \%, 6.3 \%$ and $10.7 \%, 7.9 \%, 6.4 \%$, respectively. This demonstrated that BIE for AFP measurement has excellent precision. Accuracy was analyzed by spiked recovery test [22] The recovery rates were from 93.0 to $106.5 \%$, and the detailed information was shown in Table 2. The selectivity of the BIE was evaluated with several substances including three tumor markers (CEA, CA19.9, and CA 242), two common proteins (human serum albumin, HSA; fibrinogen, Fib), the mixture of HSA and Fib and PBS. The results (Fig. 6.) demonstrated that cross reaction rates of these tested were no more than 5.2\% compared with the detection signal of AFP (with the median concentration $100.0 \mathrm{ng} / \mathrm{ml}$ ), which indicated BIE has an acceptable selectivity.

\subsection{Preliminary application}

Biomarker for cancer identification, sensitivity is the percent correct on the actually diseased population which is so-called truepositive fraction (TPF) and specificity is the percent correct on the

Table 1

The intra-slide and inter-slide reproducibility of BIE for AFP analysis.

\begin{tabular}{lrrrrrr}
\hline & \multicolumn{2}{l}{$\begin{array}{l}\text { Intra-slide } \\
\text { reproducibility }\end{array}$} & \multicolumn{4}{l}{$\begin{array}{l}\text { Inter-slide } \\
\text { reproducibility }\end{array}$} \\
\hline Sample & 1 & 2 & 3 & 1 & 2 & 3 \\
$\mathrm{~N}$ & 8 & 8 & 8 & 5 & 5 & 5 \\
Mean concentrations $(\mathrm{ng} / \mathrm{ml})$ & 42.9 & 85.1 & 134.2 & 45.7 & 86.2 & 129.5 \\
Standard deviation & 4.4 & 5.6 & 8.4 & 4.9 & 6.8 & 8.3 \\
CV\% & 10.3 & 6.6 & 6.3 & 10.7 & 7.9 & 6.4
\end{tabular}

Table 2

AFP added and detected in the recovery tests.

\begin{tabular}{lcccc}
\hline AFP $(\mathrm{ng} / \mathrm{mL})$ & \multicolumn{3}{c}{} & \multirow{2}{*}{ Recovery rate $(\%)$} \\
\cline { 1 - 3 } Known & Added & Expected & Founded & \\
\hline 21.1 & 20.0 & 41.1 & 43.76 & 106.5 \\
21.1 & 40.0 & 61.1 & 56.56 & 93.0 \\
21.1 & 80.0 & 101.1 & 96.20 & 97.2 \\
21.1 & 120.0 & 141.1 & 138.9 & 98.4 \\
\hline
\end{tabular}

actually nondiseased population which is so-called true-negative fraction (TNF). Accordingly, false-positive fraction (FPF) is the percent incorrect on the actually nondiseased population and false-negative fraction (FNF) is the percent incorrect on the actually diseased population. Their calculations can be found in literature [23]. Different cut-off values lead to multiple pairs of TPF and FPF values. The TPF minus FPF equals to Youden's index which indicates maxima diagnostic value. Generally, when Youden's index reaches maxima, the corresponding variation in detected signal is taken as the best cutoff point. A receiver operating characteristic curve (ROC) can be generated when TPF served as y coordinates and FPF as $\times$ coordinates is plotted [24].

Total 147 clinic samples including 100 healthy samples and 47 patient samples were detected by BIE. Discrete grayscale above the capture antibody control [13] was used as various cut-off level to obtain TP, FN, TN and FP when pathology test was used as reference criterion. The TPF, FPF and Youden's index were then calculated, as shown in Table 3. Obviously, the best cut-off point is 17.0 and its corresponding mass concentration is $28.6 \mathrm{ng} / \mathrm{mL}$, where the sensitivity is 0.72 and the specificity is 0.94 . Grayscale changes $(\Delta G)$ from clinic samples were analyzed in Table 4 . The range of $\Delta G$ was $0.53-$ 38.5 for healthy group and $0.27-132.5$ for patient group, respectively. Mean $\Delta \mathrm{G}$ was $8.8 \pm 7.0$ for healthy group and $46.2 \pm 37.2$ for patient group. By statistical test, the $\Delta \mathrm{G}$ in patient group has significant difference with healthy group $(\mathrm{F}=142.34, \mathrm{P}<0.01)$. We further substantiated the diagnosis performance for HCC by calculating area under ROC with the help of cut-off value initially established and 0.89 (95\% $\mathrm{CI}=0.87-0.98, \mathrm{P}<0.01)$ was obtained. As the range of area under ROC is normally among $0.5-1.0$, it validated the potential diagnosis value.

\subsection{Comparison of methodologies}

The detection results of 47 clinic samples using BIE were compared with that of ECLIA which was often taken as a gold standard in clinical application [25] and were shown in Fig. 7. There was high correlation

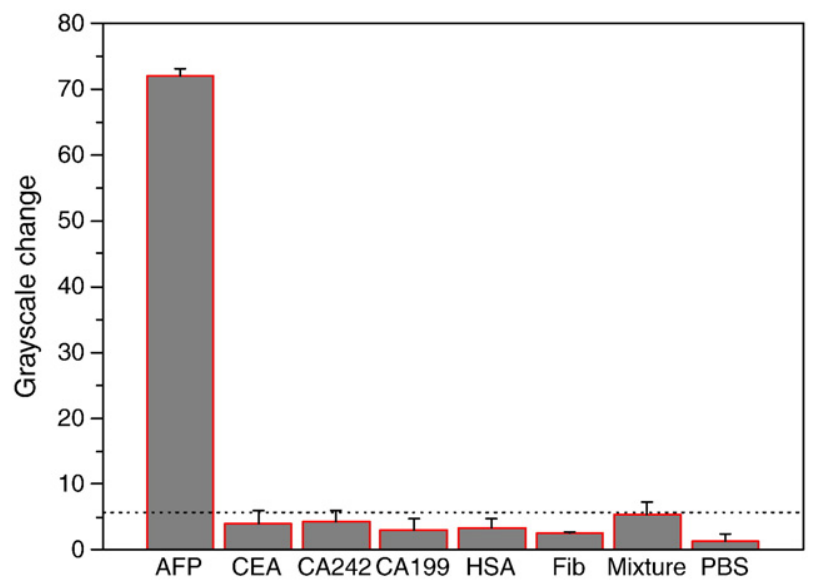

Fig. 6. Selectivity was evaluated by AFP $(100.0 \mathrm{ng} / \mathrm{ml})$, CEA $(200.0 \mathrm{ng} / \mathrm{ml})$, CA2 242 $(200.0 \mathrm{ng} / \mathrm{ml})$, CA19-9 $(200.0 \mathrm{U} / \mathrm{ml}), \mathrm{HSA}(1.0 \mathrm{ug} / \mathrm{ml}), \mathrm{Fib}(1.0 \mathrm{ug} / \mathrm{ml})$, their mixture and PBS. 
Table 3

Cut-off values for AFP detection. The $\Delta$ Grayscale $(\Delta G)$ for AFP detection is above the blank control of capture antibody. TPF is true positive fraction; FPF is false positive fraction; Positive likelihood ration is TPF divided by FPF; Youden's index is TPF minus FPF. The cut-off value is indicted by the boldfaced italics.

\begin{tabular}{lllll}
\hline$\triangle G$ & TPF & FPF & Positive likelihood ratio & Youden's index \\
\hline 1 & 0.96 & 0.94 & 1.02 & 0.02 \\
2 & 0.91 & 0.86 & 1.06 & 0.05 \\
3 & 0.89 & 0.78 & 1.14 & 0.11 \\
4 & 0.88 & 0.70 & 1.26 & 0.18 \\
5 & 0.86 & 0.64 & 1.34 & 0.22 \\
6 & 0.86 & 0.56 & 1.55 & 0.30 \\
7 & 0.86 & 0.50 & 1.72 & 0.36 \\
8 & 0.82 & 0.42 & 1.95 & 0.40 \\
9 & 0.82 & 0.38 & 2.16 & 0.44 \\
10 & 0.81 & 0.36 & 2.25 & 0.45 \\
11 & 0.78 & 0.31 & 2.52 & 0.47 \\
12 & 0.78 & 0.26 & 3.00 & 0.52 \\
13 & 0.75 & 0.23 & 3.26 & 0.52 \\
14 & 0.74 & 0.19 & 3.89 & 0.55 \\
15 & 0.73 & 0.14 & 5.21 & 0.59 \\
16 & 0.73 & 0.11 & 6.64 & 0.62 \\
$\mathbf{1 7}$ & $\mathbf{0 . 7 2}$ & $\mathbf{0 . 0 6}$ & $\mathbf{1 2 . 0 0}$ & $\mathbf{0 . 6 6}$ \\
18 & 0.70 & 0.05 & 14.00 & 0.65 \\
19 & 0.69 & 0.04 & 17.25 & 0.65 \\
20 & 0.69 & 0.04 & 17.25 & 0.65 \\
30 & 0.63 & 0.03 & 21.00 & 0.60 \\
40 & 0.56 & 0.00 & $/$ & 0.56 \\
\hline
\end{tabular}

Table 4

Data analysis for clinic samples.

\begin{tabular}{clclcccl}
\hline Group & Number & Mean \pm SD & Minimum & Median & Maximum & F value & $P$ \\
\hline $\begin{array}{c}\text { Healthy } \\
\text { group }\end{array}$ & 100 & $8.8 \pm 7.0$ & 0.53 & 7.2 & 38.5 & 142.34 & $<0.05$ \\
$\begin{array}{c}\text { Patient } \\
\text { group }\end{array}$ & 47 & $47.2 \pm 36.2$ & 0.27 & 45.6 & 132.5 & & \\
\hline
\end{tabular}

$(\mathrm{R} 2=0.9949, \mathrm{P}<0.05)$ by spearman's analysis. By calculation TP, FN, FP and TN for both patient and healthy samples, the difference between BIE and ECLIA was compared with chi-squared test $(\mathrm{F}=3.326, \mathrm{P}<0.05)$. There is a high agreement for diagnosing HCC between two methods (Kappa $=0.733, \mathrm{U}=3.21>\mathrm{U} 0.01$,). It demonstrated that the proposed method have the potential for clinic application.

\section{Conclusion}

In summary, we have developed a label-free immunoassay using biological amplification for quantitative detection of AFP with high sensitivity $(5.0 \mathrm{ng} / \mathrm{ml})$ and broad detection range $(20.0-200 \mathrm{ng} / \mathrm{ml})$. The method has high correlation and agreement with ECLIA by Spearman's and kappa analysis, respectively. The diagnosis value of the proposed method was validated by clinic sample with the help of pathology test as a reference criterion and has area under ROC (AUC) of 0.89 for HCC diagnosis. It demonstrated that it could be a potential tool for clinic application.

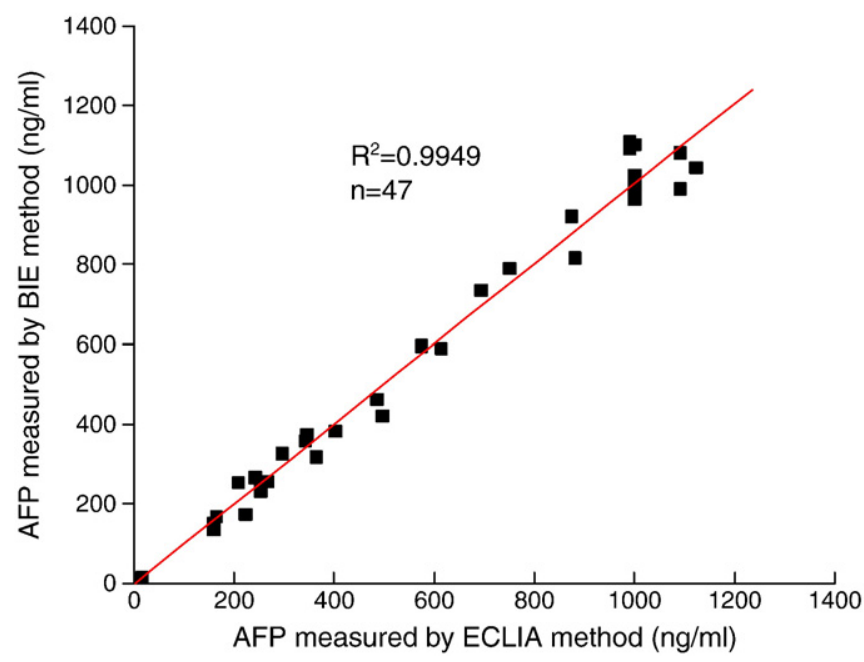

Fig. 7. Correlation between conventional ECLIA and BIE method was assayed by 47 patient samples.

\section{Acknowledgements}

The authors gratefully acknowledge financial support of the "863" goal-oriented project (2008AA02Z419), “973” project (2009CB320300), and Natural Science Foundation of China (20845003, 10804083).

\section{References}

[1] P.R. Srinivas, B.S. Kramer, S. Srivastava, Lancet Oncol. 2 (11) (2001) 698.

[2] P. Pucci, R. Siciliano, A. Malorni, Biochemistry 30 (1991) 5061.

[3] L.M. Wright, J.T. Kreikemeier, C.J. Fimmel, Cancer Detect. Prev. 31 (2007) 35.

[4] H.A. Kemp, J.S.A. Simpson, J.S. Woodhead, Clin. Chem. 27 (8) (1981) 1388.

[5] M.W. Burgett, S. Kwan, Ric. Clin. Lab. 12 (299) (1982) 301.

[6] A. Roda, P. Pasini, M. Mirasoli, E. Michelini, M. Guardigli, Trends Biotechnol. 22 (6) (2004) 295.

[7] T. Kodadek, Chem. Biol. 8/2 (2001) 105.

[8] S. Ray, G. Mehta, S. Srivastava, Proteomics 10 (2010) 731.

[9] T. Schram, A. Franquet, H. Terryn, J. Vereecken, Adv. Eng. Mater. 1 (1) (2000) 63.

[10] G. Jin, P. Tengvall, I. Lundstrom, H. Arwin, Anal.biochem 232 (1995) 69.

[11] G. Jin, R. Jansson, H. Arwina, Rev. Sci. Instrum. 67 (1996) 2930.

[12] Y.H. Meng, Y.Y. Chen, C. Qi, L. Li, G. Jin, phys. stat. sol. 5/5 (2008) 1050.

[13] C. Qi, J.-Z. Duan, Z.-H. Wang, Y.-Y. Chen, P.-H. Zhang, L. Zhan, X.-Y. Yan, W.-C. Cao, G. Jin, Biomed. Microdevices 8 (2006) 247.

[14] Qi. Cai, W. Zhu, Y. Niu, H.G. Zhang, G.Y. Zhu, Y.H. Meng, S. Chen, G. Jin, J. Viral Hepat. 16 (2009) 822.

[15] C. Qi, X.-S. Tian, S. Chen, J.-H. Yan, Z. Cao, K.-G. Tian, G.F. Gao, G. Jin, Biosens. Bioelectron. 25 (6) (2010) 1530.

[16] J.W.A. Findlay, W.C. Smith, J.W. Lee, G.D. Nordblom, I. Das, B.S. DeSilva, M.N. Khan, R.R. Bowsher, J. Pharm. Biomed. Anal. 21 (2000) 1249.

[17] G. Jin, Phys. Stat. Sol. (2008) 810, (a) 205/4.

[18] M. Stenberg, H. Nygren, J. Phys. Colloques 44 (1983) 83.

[19] Y. Chen, Y. Meng, G. Jin, Appl. Opt. 46 (35) (2007) 8475.

[20] Z.-H. Wang, G. Jin, J. Immunol. Meth. 285 (2004) 237.

[21] H.I. Yuji, Teramura analytical Biochem. 365 (2007) 201.

[22] N.R.L.H.W. Roh, Y.H. Cho, J.B. Jung, H.N. Chung, W.S. Yang, W.K. Lee, H.K. Lee, G.H. Ryu, Curr. Appl Phys. 261 (2006).

[23] D.T. burns, K. Danzer, A. Townshend, Pure Appl. Chem. 74/11 (2002) 2201.

[24] R.F. Wagner, C.E. Metz, G. Campbell, Acad. Radiol. 14 (6) (2007) 723.

[25] M.H. Zweig, G. Campbell, ClinChem 39/4 (1993) 561. 